\title{
Economicidade de despesas mediante aceitação de tecnologia: Análise da adoção de sistema eletrônico informatizado em instituições públicas
}

Expense savings through technology acceptance: Analysis of the adoption of electronic systems in public institutions

Ahorro de gastos a través de la aceptación de tecnología: Análisis de la adopción de sistemas electrónicos en instituciones públicas

\author{
Saulo Silva Lima Filho \\ ORCID: https://orcid.org/0000-0003-2199-8386 \\ Universidade Federal do Paraná, Brasil \\ E-mail: sauloslfilho@gmail.com \\ Blênio Cezar Severo Peixe \\ ORCID: https://orcid.org/0000-0001-8271-0628 \\ Universidade Federal do Paraná, Brasil \\ E-mail: bleniocsp@gmail.com
}

\begin{abstract}
Resumo
O presente estudo busca analisar a economicidade de despesas em instituições públicas por meio da Aceitação e Uso de tecnologia na informatização de processos eletrônicos. Nesse sentido, a Teoria de Aceitação e Uso de Tecnologia (UTAUT) é utilizada para explicar adesão a um sistema processual de tramitação eletrônica, que substitui papéis e demais materiais de escritório para melhorar o desempenho decorrente da economia de recursos. Trata-se de pesquisa exploratória, descritiva e explicativa quanto ao objetivo, realizada mediante análise documental com uma abordagem quantitativa. Para atingir o objetivo proposto, foram levantadas despesas antes e depois da implementação do sistema eletrônico informatizado em 94 órgãos do poder executivo federal, entre os anos de 2011 a 2020. A partir da construção de duas hipóteses de pesquisa, as análises utilizaram o teste de Wilcoxon para verificar a diferença na execução de despesas com o implemento do sistema, mas também Análise Fatorial combinada com o teste Kruskall-Wallis para identificar uma possível mudança de comportamento sistêmico nos custos advinda do uso de sistema eletrônicos. Os resultados apontam que, somente a adoção ao sistema pode não conferir argumentos suficientes para adesão à tecnologia. Contudo, a partir do componente teórico, os argumentos de agilidade, facilidade de uso e segurança da informação são pertinentes na atuação governamental para o processo de tomada de decisão dos gestores.

Palavras-chave: Teoria de aceitação e uso de tecnologia; Economicidade de despesas; Sistema eletrônico informatizado em instituições públicas.
\end{abstract}

\begin{abstract}
This study seeks to analyze the cost savings in public institutions through the Acceptance and Use of technology in the computerization of electronic processes. In this sense, the Theory of Acceptance and Use of Technology (UTAUT) is used to explain adherence to a procedural system of electronic processing, which replaces paper and other office supplies to improve performance resulting from resource savings. It is an exploratory, descriptive and explanatory research as to the objective, carried out through document analysis with a quantitative approach. To achieve the proposed objective, expenses were raised before and after the implementation of the computerized electronic system in 94 federal executive branches, between 2011 and 2020. Based on the construction of two research hypotheses, the analyzes used the test of Wilcoxon to verify the difference in the execution of expenses with the system implement, but also Factor Analysis combined with the Kruskall-Wallis test to identify a possible systemic behavior change in costs arising from the use of electronic systems. The results show that the adoption of the system alone may not provide sufficient arguments for adherence to the technology. However, based on the theoretical component, the arguments of agility, ease of use and information security are relevant in government action for the decision-making process of managers.

Keywords: Theory of acceptance and use of technology; Expense economics; Electronic system computerized in public institutions.
\end{abstract}

\section{Resumen}

Este estudio busca analizar el ahorro de costos en las instituciones públicas a través de la Aceptación y Uso de tecnología en la informatización de procesos electrónicos. En este sentido, la Teoría de Aceptación y Uso de Tecnología (UTAUT) se utiliza para explicar la adherencia a un sistema procedimental de procesamiento electrónico, que reemplaza el papel 
y otros insumos de oficina para mejorar el desempeño derivado del ahorro de recursos. Se trata de una investigación exploratoria, descriptiva y explicativa del objetivo, realizada a través del análisis documental con enfoque cuantitativo. Para lograr el objetivo propuesto, se plantearon gastos antes y después de la implementación del sistema electrónico computarizado en 94 poderes ejecutivos federales, entre los años 2011 a 2020. Con base en la construcción de dos hipótesis de investigación, los análisis utilizaron la prueba de Wilcoxon para verificar la diferencia en la ejecución de gastos con el sistema implementado, pero también Análisis Factorial combinado con la prueba de Kruskall-Wallis para identificar un posible cambio de comportamiento sistémico en los costos derivado del uso de sistemas electrónicos. Los resultados muestran que la adopción del sistema por sí sola puede no proporcionar argumentos suficientes para la adhesión a la tecnología. Sin embargo, con base en el componente teórico, los argumentos de agilidad, facilidad de uso y seguridad de la información son relevantes en la acción gubernamental para el proceso de toma de decisiones de los gerentes.

Palabras clave: Teoría de aceptación y uso de tecnología; Economía de gastos; Sistema electrónico informatizado en instituciones públicas.

\section{Introdução}

O uso de tecnologia tem sido objeto de estudo sob diversas perspectivas. Sobretudo pelas distintas condições em que seus usuários aceitam determinadas ferramentas para facilitar e agilizar o acesso aos processos eletrônicos por meio do uso de tecnologia. Diante desse dilema, a teoria proposta por Venkatesh, Morris, Davis e Davis (2003), denominada Teoria de Aceitação e Uso de Tecnologia, em inglês conhecida por UTAUT, aponta alguns aspectos que podem aproximar a tecnologia de seus usuários.

Um dos argumentos utilizados pelos autores (Venkatesh; Morris; Davis; Davis, 2003), remete à possibilidade de ampliação no desempenho, seja por processos mais otimizados, bem como pela possibilidade de redução nos insumos necessários para condução das atividades (Gruzd; Staves; Wilk, 2012). Trata-se de uma condição que denota um aumento na produtividade afetando as condições de competitividade da instituição.

Percebe-se portanto, que a busca por um sistema eletrônico informatizado responsável pela tramitação processual no âmbito do setor público possui acolhimento nesse mesmo amparo teórico. Todavia, o argumento sobre a economicidade de despesas ainda é questionável, frente ao vultoso volume de gastos geradas em toda a administração pública, que poderia diluir os benefícios advindos com o uso de sistema eletrônico informatizado. Sob esta perspectiva, o presente estudo busca analisar a economicidade de despesas em instituições públicas por meio da Aceitação e Uso de tecnologia na informatização de processos eletrônicos.

Diante deste objetivo proposto, o estudo se baseia na perspectiva da Teoria de Aceitação e Uso de Tecnologia para analisar o ganho de desempenho com aumento da eficiência técnica e operacional na economia de despesa. E utiliza como um argumento recorrente para que instituições públicas formalizem adesão por meio da aceitação do uso da tecnologia de sistema eletrônico informatizado. Isto é, as demais perspectivas apontadas por (Venkatesh et al., 2003) ainda representam argumentos relevantes, independentes dos resultados desta análise. O que não deprecia os benefícios advindos com a utilização do sistema eletrônico informatizado.

Feitos esses apontamentos, acentua-se que a presente pesquisa se mostra relevante sob diferentes perspectivas. Seja pelo ponto de vista acadêmico que utiliza um arcabouço teórico, para testar uma aplicação na prática, que pode ser utilizada na divulgação da adesão ao sistema eletrônico informatizado em instituições públicas para dar visibilidade na conquista de novos usuários e agilidade na criação e tramitação de processos. 
Por outro lado, há uma perspectiva pragmática que não pode ser desprezada, tendo em vista o fator da economicidade das despesas públicas por meio do aumento da eficiência técnica e operacional, que é decisivo na gestão em um ambiente de recursos escassos, sob constante fiscalização da aplicação de recursos públicos. O uso otimizado de recursos públicos é almejado em todas as instâncias governamentais de forma permanente por parte dos gestores públicos.

Ademais, também é percebida uma relevância social, a partir do momento que aborda uma ferramenta que permite dar maior transparência e agilidade na tramitação de informação útil para tomada de decisão. Além de verificar se é cabível a justificativa de melhoria nos indicadores de desempenho, por meio do aumento da eficiência técnica e operacional, no uso de recursos públicos limitados.

O estudo foi estruturado de modo a apresentar a contextualização teórica aplicável à Teoria de Aceitação e Uso de Tecnologia, como também à reforma gerencial que alterou a condução de procedimentos no setor público. Face a esse referencial teórico, discorre-se sobre a metodologia adotada e análises correspondentes dos resultados do estudo.

\section{Referencial Teórico}

$\mathrm{Na}$ abordagem do referencial teórico destaca-se o estudo da teoria de aceitação e uso de tecnologia, estudos anteriores sobre a teoria de aceitação e uso de tecnologia, economicidade de recursos públicos e construção das hipóteses de pesquisa

\subsection{Teoria de Aceitação e Uso de Tecnologia}

A aceitação do uso de tecnologia tem recebido atenção desde a adoção de mecanismos informatizados à rotina das organizações. De modo concomitante, acadêmicos também passaram a se interessar pela forma como a utilização dessas novas ferramentas afeta as atitudes dos usuários e quais seriam as resistências a esse novo modo de trabalho (Bhatiasevi, 2016). Diante de todas as relações existentes entre os usuários e as ferramentas de tecnologia, diferentes teorias surgiram e passaram a explicar conceitos e comportamentos. Entretanto, tais teorias não eram complementares e havia lacuna para criação de um constructo que as relaciona-se e sintetiza-se suas relações em um modelo unificado (Bhatiasevi, 2016).

A partir da integração dos fragmentos de oito modelos teóricos surge a Teoria Unificada de Aceitação e Uso de Tecnologia (Unified Theory of Acceptance and Use of Technology - UTAUT), a qual foi capaz de adicionar aos modelos existentes a possibilidade de predição sobre intenções e comportamentos (Venkatesh; Morris; Davis; Davis, 2003). Para tanto, foram definidos parâmetros moderadores decorrentes das características dos usuários de tecnologia como a idade, gênero, experiência e a disposição para uso das ferramentas.

O constructo proposto pela teoria parte de conceitos essenciais para explicar a aceitação e o uso de ferramentas de tecnologia. Segundo Venkatesh et al., (2003) a expectativa de desempenho, a expectativa de esforço, a influência social e demais condições facilitadoras seriam moderadas pelas características dos usuários e permitiram com razoável confiabilidade explicar como os usuários recebem ou repelem a tecnologia, bem como sua predisposição ao uso.

Os estudos de Venkatesh et al., (2003) deram a oportunidade para pesquisadores aplicarem as relações propostas no modelo teórico em diferentes aplicações e contextos. Como no caso de Bhatiasevi (2016) ao testar hipóteses de conceitos ligados ao desempenho, expectativa de esforço, facilidades, dentre outros, frente ao comportamento dos usuários de mobile banking. Isto é, dentre outros fatores, torna-se possível explicar e predizer o comportamento de usuários de tecnologia conforme sua expectativa de desempenho. Nesse contexto, acredita-se que o usuário avalie a quão útil determinada tecnologia pode ser e qual o impacto em sua produtividade (Gruzd; Staves; Wilk, 2012). A aceitação de tecnologia a partir desse conceito convence o usuário dos benefícios advindos que envolvem a percepção de vantagem competitiva e melhores resultados (Magsamen-Conrad; Upadhyaya; Joa; Dowd, 2015). 
Ademais, o modelo teórico criado por Venkatesh (et al., 2003) atribui ao construto "condições facilitadoras" a propriedade de indicar ao usuário se possui os recursos necessários para usufruir determinada tecnologia. Diante dessa reflexão, a aceitação ocorre ao verificar que a ferramenta tecnológica é compatível com o uso esperado e com o conhecimento preciso para extrair seus benefícios (Gruzd; Staves; Wilk, 2012).

Por sua vez, também é preciso considerar que o constructo destinado à influência social trata da influência que outras pessoas possuem sobre o comportamento dos usuários que utilizam a tecnologia. Ou seja, pessoas consideradas distintas ou de relevância ao usuário podem determinar sua predisposição ao uso. A UTAUT assume que a influência social possui um efeito relevante sobre a intenção do usuário em aceitar a tecnologia (Gruzd; Staves; Wilk, 2012).

Entretanto também são verificados indícios contrários à aceitação e uso de tecnologia. Trata-se de fatores que podem criar rejeição, especialmente quando relacionados com as características de determinados usuários. O modelo sugere diferenças nas percepções entre pessoas de diferentes idades, gêneros ou mesmo experiência com tecnologia. Deste modo, em algumas circunstâncias o próprio usuário agrega condições de rejeição que dificultam a implementação de tecnologia (Magsamen-Conrad et al., 2015).

É o caso das características de trabalho no setor público, em que também são verificados indícios que podem comprometer a aceitação e uso de ferramentas tecnológicas. Em particular as condições políticas, procedimentos rígidos e a falta de incentivos podem criar empecilhos e desestimulem a aceitação pelos servidores públicos (Fulton; Farias; Alfinito; Almeida, 2018).

\subsection{Estudos Anteriores sobre a Teoria de Aceitação e Uso de Tecnologia}

A UTAUT como mencionado possui aplicação em diversos contextos. Tanto que pesquisas acadêmicas a utilizam para verificar como os usuários aceitam e utilizam recursos tecnológicos. É o caso de Sherer e Teo (2019) que buscam descrever as intenções do uso de tecnologia por professores a partir de um constructo gerado por uma das teorias que compõem a UTAUT. Seu modelo permitiu explicar aproximadamente $1 / 3$ das intenções dos professores em usar tecnologia, considerando suas publicações e pela experiência do docente.

Por sua vez, Zhao, Ni e Zhou (2018) buscaram desenvolver um quadro sobre a adoção de serviços de saúde móveis individuais, utilizando o moderador de acordo com a idade. Mediante análises de correlação, os autores identificaram, dentre outros fatores, que a utilidade percebida e a facilidade de uso influenciam a intenção de comportamento, especialmente em relação aos usuários de meia idade e idosos.

Ao se deparar com os vultosos gastos em processos públicos e serviços online, frente à busca por governos mais eficientes e voltados à realização de serviços públicos em países da África, Mosweu e Bwalya (2018) investigaram os fatores determinantes que influenciam a adesão e uso do sistema de gerenciamento do fluxo de trabalho de documentos para registros eficazes de gestão de informações dentro do Ministério de Investimento, Comércio e Indústria. Os resultados foram limitados em face aos aspectos subjetivos do comportamento humano, visto que $45 \%$ da variância não pode ser explicada, segundo os autores, por conta da variabilidade de contextos que indivíduos em países em desenvolvimento estão sujeitos.

Em outra perspectiva, especialmente ao considerar o crescimento exponencial nos dados disponíveis para análise, Cabrera-Sanchez e Villarejo-Ramos (2019) investigam as razões pelas quais empresas deixam de usar recursos da data analytics para otimizar seus negócios. Por meio da UTAUT, os resultados apontam o ceticismo de alguns usuários com os resultados que podem ser alcançados, além disso, também é discutido a dificuldade de implantação visto a infraestrutura instalada que é demandada. 
Outro tema recente e abordado nos estudos anteriores, trata do uso do armazenamento em cloud computing. Para tanto, Palos-Sanchez, Reyes-Menendez e Saura (2019) realizaram uma revisão sistemática de literatura. Os resultados indicam que os estudos sobre o tema têm abordado justamente a aceitação e uso de tecnologias, seja pela UTAUT e demais teorias correlatas.

No que se refere ao uso da teoria em estudos da área pública, há que se mencionar o trabalho de Carrera-Mora, Ovando, Villafuerte e Parada (2019) ao determinar a relação entre a perspectiva de eficiência cidadã e o comportamento de uso do egoverno municipal. Segundo os autores, a perspectiva de eficiência do cidadão é um fator que influencia o uso do e-governo, incentivando a adesão aos meios digitais. Ademais, o estudo propõe que fatores como o aumento da confiança na internet, a facilidade de uso e a familiaridade com a tecnologia, dentre outros, auxiliam na aceitação e uso de ferramentas de e-governo.

\subsection{Economicidade de Recursos Públicos}

No que se refere à economicidade de recursos, cumpre esclarecer que a busca pela eficiência técnica e operacional na administrativa, inerente à reforma gerencial, apregoa aspectos como agilidade e segurança nas funções administrativas, visto que adota práticas privadas na gestão e controle da administração pública (Matias-Pereira, 2018). Diante disto, nota-se que iniciativas como a informatização de procedimentos, estão alinhados com a gestão pública contemporânea, que incentiva a busca por desempenho por meio da eficiência técnica e operacional, gerando resultados e tendo o foco no cliente, neste caso, a sociedade.

Assume-se portanto, que o mecanismo de tecnologia que fornece agilidade, segurança e economia de recursos está condizente com as expectativas oriundas da UTAUT. Sobretudo por conta do aumento no desempenho decorrente da busca por melhores opções de tramitação de processos. De fato, o movimento gerencial proposto pela Nova Administração Pública (NAP) redimensiona o modo como o ente governamental interpreta sua relação com a sociedade em diferentes perspectivas, inclusive na economicidade de recursos mediante procedimentos mais céleres e que consomem menos recursos (Steccolini, 2019).

Vale salientar que o Sistema Eletrônico de Informações (SEI), assim como outros sistemas de informações ganham atenção à medida que promovem benefícios à instituição os quais, pela perspectiva de Purnama, Cahyono e Wijaya (2020) tratam de implementos de governança em tecnologia. Portanto, ao trazer à discussão as razões para aceitação e uso de tecnologia (Venkatesh et al., 2003) e a busca pela eficiência técnica e operacional, é possível assumir que, a economicidade de despesas é um fator que motiva as organizações a se inovarem e trazerem ferramentas tecnológicas para a operacionalização online de processos por meio da agilidade das transações eletrônicas e com a consequente redução de custos.

\subsection{Construção das Hipóteses de Pesquisa}

A partir do referencial teórico, assume-se que a redução de despesas públicas é um importante trunfo pleiteado pelos sistemas eletrônicos informatizados. Sobretudo em circunstâncias de baixa atividade econômica quando a administração pública precisa criar mecanismos que tragam agilidade, segurança e economia de recursos públicos. Ao avaliar essa demanda, é possível aceitar que o uso de ferramentas tecnológicas encontram amparo sob a UTAUT (Venkatesh et al., 2003).

Contudo, a aceitação de tecnologia possui critérios teóricos a serem observados. As diferentes condições de trabalho e as distinções de aptidão tecnológica entre usuários podem alterar a forma como a aceitabilidade ocorre nas diversas unidades administrativas (Magsamen-Conrad et al., 2015). Em virtude dessas possíveis resistências, a economicidade de recursos, a possibilidade de ganho de desempenho e ainda o incentivo gerencial decorrente de estímulo gerado pelos gestores públicos, podem impulsionar a adesão ao uso de tecnologia (Venkatesh et al., 2003).

Portanto, ao considerar que a busca por melhor desempenho faz parte do escopo apontado pela UTAUT (Gruzd; Staves; Wilk, 2012), a pesquisa investiga se a aceitação de tecnologia na informatização de processos eletrônicos oferece economicidade às instituições públicas. Para tanto, inicialmente é levantada a seguinte hipótese de pesquisa. 
Hipótese Alternativa $\boldsymbol{H}^{\boldsymbol{I}}$ - A implementação de um sistema informatizado de tramitação eletrônica oferece maior economicidade de recursos públicos.

Saliente-se que dentre os argumentos de recusa aos meios tecnológicos no encaminhamento processual, a economicidade de despesas deve se sobrepor e apontar os benefícios que uma tramitação ágil com considerável economia de despesas pode trazer ao ente público. Especialmente em ambientes com forte resistência às mudanças e que possuam empecilhos no implemento de tecnologia, a possibilidade de agilidade e ganho de desempenho também precisa ser destacada (Venkatesh et al., 2003; Magsamen-Conrad et al., 2015).

Em meio a fatores que podem rejeitar o uso do sistema eletrônico pelos órgãos públicos, há ainda que se ressaltar a possibilidade da economia de despesas ocorrer de maneira indistinta entre os diferentes grupos de Naturezas de Despesas. Isto é, se a redução nos gastos atingiu de modo equânime os demais subelementos de despesas. Para tanto, é preciso considerar despesas não correlatas, mas que guardem proximidade com os itens que mais recebem impacto com a informatização de sistemas. Como apontado pela literatura, as despesas com Material de Expediente são aquelas que nitidamente estão mais relacionadas à adoção a sistemas de informação, dada a mudança de hábitos que torna desnecessário adquirir itens como papel e demais materiais de escritório (Giacomoni, 2019; ME, 2020). Portanto, é averiguado se a eventual economia de recursos é saliente a este nicho de despesas, ou se ocorre de forma sistêmica e acabam não sendo notadas diferenças nos grupos de redução de despesa pública.

Hipótese Alternativa $\boldsymbol{H}^{2}$ - A economicidade de recursos, advinda com a adoção de um sistema informatizado de tramitação eletrônica, ocorre de modo distinto entre as naturezas de despesas.

Posto isso, acentua-se que a confirmação das hipóteses de pesquisa permitiria concluir que a aceitação de tecnologia no âmbito público aufere benefícios econômicos no uso dessas ferramentas. Além de se tornar evidente que seus reflexos estão nitidamente ligados ao uso de sistemas de tecnologia da informação.

A rejeição das hipóteses assume que a aceitação da tecnologia ainda não reflete os benefícios econômicos esperados por meio de dispêndios nas contas públicas. Um contraponto aos anseios apresentados pelos órgãos governamentais, como o próprio Ministério da Economia (ME, 2020) que esperam pela relevante redução de custos a partir da redução no uso de materiais de expediente. Cabe frisar que a não aceitação da hipótese também representa um problema na aceitabilidade do sistema, haja vista que os argumentos teóricos apontados deixam de ser observados, em especial os benefícios de desempenho advindos com a aceitação e uso da tecnologia.

\section{Metodologia}

O presente estudo foi classificado como exploratório, descritivo, explicativo e quanto aos procedimentos é documental, além de utilizar uma abordagem quantitativa para análise dos resultados. Nesse sentido, visando responder às hipóteses de pesquisa, a análise dos resultados é conduzida em duas etapas. Primeiramente é analisada a redução de despesas nos períodos antes e após a implantação de um sistema eletrônico para criação e tramitação de processos. Para então aprofundar a investigação a partir da possibilidade de economia de despesas ter ocorrido de modo sistêmico.

Há que se salientar que são notadas diversas iniciativas similares para tornar os processos mais ágeis mediante sua informatização. Entretanto, no presente estudo, foi utilizado como proxy o Sistema Eletrônico de Informações (SEI), oriundo de uma iniciativa conjunta entre diversos órgãos e entidades da administração pública, os quais buscaram uma ferramenta de infraestrutura pública para criação e tramitação eletrônica de processos por meio de documentos administrativos. 
Sob a coordenação do então Ministério do Planejamento, Desenvolvimento e Gestão, o SEI busca a melhoria no desempenho de atividade na administração pública, mediante ganhos com agilidade, produtividade, transparência, satisfação dos usuários e redução de custos. Segundo informações do próprio ministério, a intenção do projeto é oferecer uma ferramenta flexível para se adaptar à necessidade de órgãos e entidades de toda a administração pública, independente do ramo de atividade (ME, 2020).

Os benefícios apontados pela adoção ao SEI são consoantes às sustentações feitas pela UTAUT, sobretudo quanto à economia de recursos, ganhos de agilidade e segurança da informação. Entretanto, Nowak (2018) ainda defende que o sistema adotado deve promover uma interface amigável, que traz consigo versatilidade e facilidade no uso, além de propriedades como aperfeiçoamento das etapas de trabalho. Logo, percebe-se a possibilidade de ganho ainda à gestão documental, sobretudo à celeridade processual, segurança e transparência das informações, mas também economicidade de despesas (Fulton et al., 2018).

É preciso frisar que, assim como aponta o Ministério da Economia (ME, 2020), o uso de sistemas informatizados para tramitação de processos não atinge de modo equânime as despesas públicas quando analisadas em suas especificidades. Ocorre que de acordo com a classificação por Natureza de Despesa (Giacomoni, 2019), existem despesas de capital, em grupos não relacionados com despesas correntes, que não são objetos de aplicação direta, ou ainda não se referem à aquisição de material de consumo e que não se relacionam intimamente com os objetos de despesas substituídos pelos sistemas de tramitação processual eletrônico.

Ao analisar as categorizações disponíveis, percebe-se que a economicidade advinda com a utilização do sistema pode ser ligada às despesas classificadas pela rubrica 3.3.90.30.16 (Materiais de Expediente). Mas precisaria ser expressiva e vultosa o suficiente para promover uma redução relevante nas despesas públicas (GIACOMONI, 2019; ME, 2020). Os procedimentos de pesquisa estão descritos no Quadro 1.

Quadro 1: Procedimentos de Análise.

\begin{tabular}{|c|c|c|c|c|}
\hline Hipótese & Variáveis & $\begin{array}{l}\text { Ferramenta de } \\
\text { Análise }\end{array}$ & Objetivo & Referência \\
\hline - - & -- & $\begin{array}{l}\text { Estatísticas } \\
\text { Descritivas }\end{array}$ & $\begin{array}{l}\text { Descrever o comportamento das despesas } \\
\text { antes e após a implantação do sistema }\end{array}$ & Fávero e Belfiore (2017) \\
\hline \multirow{2}{*}{$\boldsymbol{H}^{1}$} & \multirow{2}{*}{$\begin{array}{l}\text { Classificação por } \\
\text { ND }^{1}-30.16 \\
\text { Material de } \\
\text { Expediente }\end{array}$} & $\begin{array}{c}\text { Teste de } \\
\text { Normalidade e } \\
\text { Homogeneidade }\end{array}$ & $\begin{array}{l}\text { Identificar os pressupostos para utilização de } \\
\text { testes paramétricos de diferenças de médias }\end{array}$ & $\begin{array}{l}\text { Gross e Ligges (2015); } \\
\text { Fávero e Belfiore (2017) }\end{array}$ \\
\hline & & $\begin{array}{l}\text { Teste } t \text { ou de } \\
\text { Wilcoxon }\end{array}$ & $\begin{array}{l}\text { Verificar a diferença entre grupos dois anos e } \\
\text { dois anos após a implantação do sistema }{ }^{2}\end{array}$ & Fávero e Belfiore (2017) \\
\hline \multirow{3}{*}{$H^{2}$} & \multirow{3}{*}{$\begin{array}{l}\text { Classificação por } \\
\text { ND }^{1}-30.16 \\
\text { Material de } \\
\text { Expediente e } \\
\text { outras } 60 \text { rubricas } \\
\text { de materiais de } \\
\text { consumo }\end{array}$} & Análise Fatorial & $\begin{array}{l}\text { Criar fatores a partir do agrupamento das } 61 \\
\text { variáveis observáveis }\end{array}$ & $\begin{array}{l}\text { Hair, Black, Babin, Anderson } \\
\text { e Tatham (2009); Maier } \\
\text { (2015); Ossani e Cirillo (2020) }\end{array}$ \\
\hline & & $\begin{array}{c}\text { Teste de } \\
\text { Normalidade } \\
\text { Multivariada }\end{array}$ & $\begin{array}{l}\text { Identificar os pressupostos para utilização de } \\
\text { testes paramétricos de diferenças entre grupos }\end{array}$ & $\begin{array}{l}\text { Korkmaz, Goksuluk, } \\
\text { Zararsiz (2014) }\end{array}$ \\
\hline & & $\begin{array}{c}\text { Análise de } \\
\text { Variância ou teste } \\
\text { de Kruskall-Wallis }\end{array}$ & $\begin{array}{l}\text { Verificar a diferença entre a variância entre os } \\
\text { subelementos de despesas }\end{array}$ & Fávero e Belfiore (2017) \\
\hline
\end{tabular}

Nota: ${ }^{1}$ ND - Natureza de Despesa; ${ }^{2}$ Conforme metodologia adotada pelo Ministério da Economia (ME, 2020) para avaliação da economicidade advinda com o SEI em dois anos antes e dois anos após a adesão ao sistema em cada órgão analisado.

Fonte: Autores. 
Nota-se que as investigações se ocupam em analisar o conceito de "Economicidade", se dividem em duas hipóteses de pesquisa, investigadas a partir de uma mesma base de dados. Nesse sentido, a coleta se deu mediante consulta ao sistema Tesouro Gerencial (STN, 2020), que consolida as informações registradas no Sistema de Administração Financeira do Governo Federal em uma única plataforma, que possibilitou a evidenciação de todas as despesas anuais de cada unidade.

Tal base é composta por 61 variáveis contínuas, correspondentes às despesas com materiais de consumo em 94 órgãos do poder executivo federal, nos dois anos anteriores e nos dois anos posteriores à implantação do sistema eletrônico em cada órgão, perfazendo dados entre 2011 a 2020.

Como citado, o levantamento dessas variáveis utilizou como rubrica apenas despesas com material de consumo, visto a possibilidade de comparação em relação à Categoria Econômica, Grupo de Natureza da Despesa e elementos equivalentes (Giacomoni, 2019). Ademais, foi utilizada a Modalidade de Aplicação 90, dada a aplicação direta do orçamento nas atividades funcionais das instituições.

Consoante à $H^{1}$, foi verificado se as despesas executadas nos dois anos anteriores à implantação do sistema possuem variância assemelhada às despesas executadas dois anos após o uso do SEI (Fávero; Belfiore, 2017). Trata-se da mesma metodologia de análise utilizada pelo Ministério da Economia para avaliar os benefícios com o sistema eletrônico de Informações (ME, 2020).

Considerando que se busca analisar o comportamento das despesas antes e após o implemento do SEI, aplica-se o teste não-paramétrico de Wilcoxon, adequado para testar se não há diferença entre as amostras. Segundo Fávero e Belfiore (2017), o teste de Wilcoxon é uma alternativa ao teste $t$ para amostras emparelhadas e também representa uma extensão do teste dos sinais, visto que aponta a magnitude da diferença entre os pares.

Por sua vez, para análise de $H^{2}$, em virtude da complexidade em analisar de forma distinta as 61 variáveis, foi realizada a Análise Fatorial por Componentes Principais, visando representar o comportamento desse conjunto volumoso de variáveis, em uma quantidade reduzida de fatores. Trata-se de um método de extrair fatores não correlacionados, decorrentes de combinações lineares de variáveis ortogonais (Fávero; Belfiore, 2017).

Ademais, para a verificação de uma possível redução sistêmica de despesas, de acordo com a segunda hipótese de pesquisa, os fatores foram analisados entre si, na busca por semelhanças entre os subelementos de despesas. Dessa forma, refutar $H^{2}$ seria equivalente a assumir que as despesas se comportaram de maneira assemelhada. Isto é, uma eventual redução de despesa não teria ocorrido somente em determinados subelementos relacionados à utilização de um sistema informatizado.

Portanto, para verificar a proposição feita em $H^{2}$, será utilizado o teste de Kruskal-Wallis, o qual identifica se $K$ amostras independentes (em que $\mathrm{K}>2$ ) decorrem da mesma população. Como mencionado, assim como o teste Wilcoxon, trata-se de um procedimento não-paramétrico, alternativo à Análise de Variância (ANOVA), visto que não atende aos pressupostos de normalidade e homogeneidade das variâncias (Fávero e Belfiore, 2017). Todas as análises mencionadas foram realizadas no software estatístico R, versão 4.0.2 (R Core Team, 2020).

\section{Resultados e Discussão}

Nesta análise apresenta-se a estatística descritiva, testes de diferenças de médias e análise fatorial por componentes principais.

\subsection{Estatística Descritiva}

Em atenção ao protocolo de pesquisa, dá-se início pelas estatísticas descritivas, as quais buscam descrever o comportamento do dispêndio de recursos antes e após a adoção à informatização eletrônica dos processos. Logo, como demonstra a Tabela 1, o comportamento das despesas destoam em alguns pontos. 
Tabela 1: Estatísticas Descritivas.

\begin{tabular}{c|c|c|c|c|c|c|c|c}
\hline \multicolumn{2}{c}{ Min } & $\mathbf{1}^{\circ} \mathbf{Q t}$ & Média & Mediana & Desv.Pad & QV & $\mathbf{3}^{\circ} \mathbf{Q t}$ & Máx \\
\hline Antes & 1,14 & 64,02 & 128,13 & 72,69 & 116,48 & 0,91 & 251,05 & 252,87 \\
\hline Depois & 1,47 & 1,17 & $7.797,19$ & 1,64 & $10.991,81$ & 1,41 & $15.789,07$ & $23.194,09$ \\
\hline Variação & $129,39 \%$ & $1,82 \%$ & $6.085,53 \%$ & $2,26 \%$ & $9.436,50 \%$ & $155,06 \%$ & $6.289,15 \%$ & $9.172,22 \%$ \\
\hline
\end{tabular}

Nota: Valores em milhões de reais.

Fonte: Autores (2020).

Primeiramente, quanto à distância interquartílica, nota-se que os valores mínimos estão concentrados no primeiro quartil $(64,02)$, ao passo que após a implantação a concentração se volta ao terceiro quartil $(15.789,07)$. Isso aponta uma tendência de que os valores não se reduziram após a adoção ao uso do sistema eletrônico informatizado, pelo contrário, a maior parte dos valores registrados encontram-se com maiores valores.

Também foi observada uma distinção quanto aos indicadores de tendência central, dado que os valores da média e da mediana não são aproximados. Tais resultados indicam que a média pode não ser um bom estimador para refletir a centralidade da amostra. Ademais, percebe-se uma distinção entre os coeficientes de desvio padrão, notadamente maiores após a implantação do sistema eletrônico informatizado, que refletem a disparidade dos dados. Ao passo que o coeficiente de variação, não demonstra essa dispersão, visto que seu quociente é dado pela média, igualmente vultosa.

Diante desses resultados, de modo preliminar, é possível apontar que a economia de despesas não é nítida após a adoção ao instrumento de tecnologia na tramitação de processos. Saliente-se que os dados correspondem ao agrupamento de 61 subelementos de despesas, e que muitos podem não surtir o efeito decorrente da adoção a um sistema eletrônico informatizado na tramitação de processos (ME, 2020).

Como indica o Figura 1, as despesas após a adoção ao sistema foram visualmente superiores. Este resultado, apesar de merecer uma análise parcimoniosa, em virtude da combinação entre diversas rubricas de naturezas de despesas, demonstra que a almejada redução de despesa não pode, até o momento, ser utilizada como ferramenta indiscriminada de promoção ao implemento de tecnologia da informação.

A partir do contexto teórico (Venkatesh et al., 2003; Gruzd; Staves; Wilk, 2012), a aceitação e implemento do uso de tecnologia utilizam como prerrogativas, dentre outras, a vantagem competitiva advinda com a modernização e agilidade de procedimentos. Nesse contexto, não é possível aludir essa vantagem à economicidade de despesas, o que pode minar o anseio pela implementação. 
Figura 1: Comportamento das Despesas, antes e após a adoção ao uso do SEI.

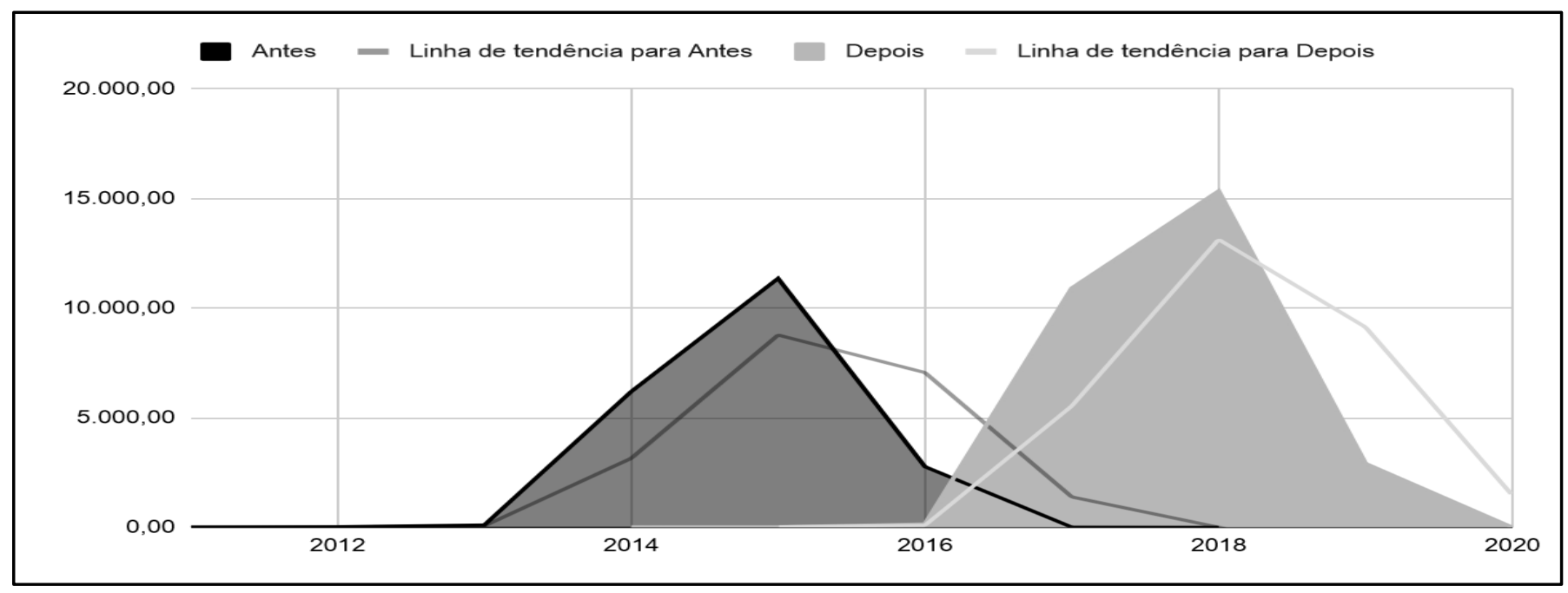

Nota: Valores em milhões de reais; Linha de tendência corresponde à média móvel de 2 períodos, consoante à metodologia adotada pelo ME (2020).

Fonte: Autores.

Por outro lado, ao analisar as despesas estritamente ligadas à redução de despesas, sobretudo àquelas que tratam de materiais de expediente, o Figura 2 apresenta indícios de uma leve redução nas despesas. Como suposto pelo próprio ME (2020), a redução de despesas é mais clara nos objetos que foram substituídos com a tramitação eletrônica de processos.

Figura 2: Elemento 16 - Despesas com Materiais de Expediente.

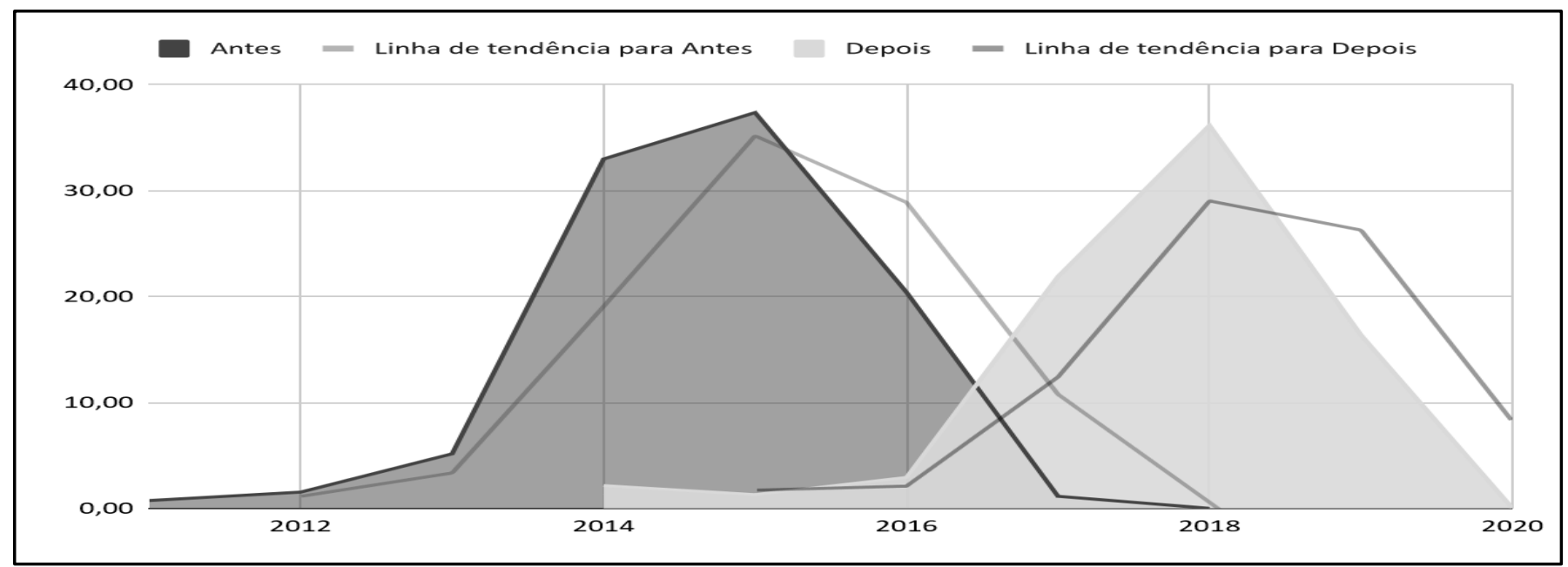

Nota: Valores em milhões de reais; Linha de tendência corresponde à média móvel de 2 períodos, consoante à metodologia adotada pelo ME (2020).

Fonte: Autores.

Entretanto, é preciso averiguar se essa eventual redução nas despesas é percebida de maneira significativa. Para tanto, foram realizados testes de diferença entre grupos que permitem identificar se o comportamento da amostra após a adoção do sistema eletrônico informatizado traduz em economicidade de despesas.

\subsection{Testes de Diferenças de Médias}

Visando identificar a possibilidade de aplicação de teste paramétricos, foi verificada a normalidade univariada (Fávero; Belfiore, 2017). Considerando que o tamanho da amostra $(n>50)$ foi aplicado o teste de Kolmogorov-Smirnov na amostra 
anterior $(\mathrm{D}=0.4622$, p-valor $<0,0000)$ e posterior à implantação do sistema $(\mathrm{D}=0.4757$, p-valor $<0,0000)$, cuja hipótese alternativa traz evidência da ausência de normalidade da amostra.

Saliente-se que não houve a necessidade de apresentar o teste de homogeneidade das variâncias, visto a quebra do primeiro pressuposto aos testes paramétricos. Diante disso, foi utilizado o teste de amostras emparelhadas de Wilcoxon (Fávero e Belfiore, 2017). Tanto os resultados para a amostra completa das 61 variáveis $(\mathrm{W}=67941$, $\mathrm{p}$-valor $=0,5236)$, quanto aqueles referentes apenas às despesas com material de expediente $(\mathrm{W}=68034$, p-valor $=0,1269)$ não sustentam a Hipótese Alternativa $H^{1}$, visto que não é percebida uma diferença significativa entre as amostras.

Como apontado anteriormente, este resultado não indica que não houve redução nas despesas com a implantação do sistema eletrônico informatizado. Apenas assegura que sua economicidade não é percebida de maneira evidente ao ponto de ser estatisticamente significativa. Além disso, a partir da UTAUT (Gruzd; Staves; Wilk, 2012; Magsamen-Conrad et al., 2015), acredita-se que a abordagem acerca da aceitação do sistema deva envolver outros parâmetros, principalmente, como a facilidade de aprendizagem, ganhos em tempo, segurança das informações, ou mesmo a transparência na tramitação processual. Todavia não é possível atribuir a economicidade como um requisito de valor que traga aceitação a esta ferramenta de tecnologia.

\subsection{Análise Fatorial por Componentes Principais}

Depois dos testes que não sustentaram a manutenção $H^{1}$, em atenção ao protocolo de pesquisa, cumpre ainda identificar a eventual distinção entre os grupos, de modo que existe uma eventual ausência de diferença entre grupos de despesas do mesmo elemento que demonstra um movimento sistêmico na execução das despesas públicas. Para isso, o agrupamento entre estes fatores busca atribuir a um seleto grupo de variáveis características que indiquem um comportamento uníssono, típico das despesas ligadas à adoção ao SEI.

Assim, inicialmente são analisados os pressupostos que indicam a adequação da amostra para realização da análise fatorial. Segundo o teste de esfericidade de Bartlett $\left(\chi^{2}(1830)=26891.739\right.$, p-valor $\left.<0,0000\right)$ e o critério de Kaiser-Meyer-Olkin $(\mathrm{KMO}=0,6697)$ a amostra está minimamente adequada para prosseguimento com a análise.

Cabe salientar que foram retiradas todas as cargas menores que 0,3, como indicado em Hair (et al., 2009). Ademais, foi realizada a rotação Varimax, para verificar o comportamento das cargas fatoriais, situação em que se obtiveram apenas 4 variáveis com cargas adequadas no critério adotado. Diante disso, optou-se pela manutenção da rotação ortogonal.

Posto isso, conforme esclarece a Tabela 2, a capacidade explicativa bidimensional por meio da variância acumulada dos autovalores é de aproximadamente 30,12\%. Valor considerado aquém do esperado, mas minimamente satisfatório para análise do conjunto das variáveis. Saliente-se que seriam necessárias 61 dimensões para explicação de $100 \%$ da variância observada.

Tabela 2: Autovalores.

\begin{tabular}{c|c|c|c}
\hline \multicolumn{1}{c|}{ Fator } & Autovalor & Variância & 18,4980 \\
\hline 1 & 11,2838 & 11,6201 & 18,4980 \\
\hline 2 & 7,0883 & 9,1592 & 30,1181 \\
\hline 3 & 5,5871 & 4,7627 & 39,2774 \\
\hline 4 & 2,9052 & 4,1667 & 44,0400 \\
\hline 5 & 2,5417 & 3,7852 & 48,2068 \\
\hline 6 & 2,3090 & 3,5296 & 51,9920 \\
\hline 7 & 2,1531 & $\ldots$ & 55,5216 \\
\hline 61 & 0,0019 & & \\
\hline
\end{tabular}

Fonte: Autores (2020). 
O levantamento criou 7 fatores, em virtude das características análogas entre as variáveis. Nesse sentido, foram consideradas despesas com materiais para laboratório, manutenção de máquinas e equipamentos, Tecnologia de Informação e Comunicação, materiais biológicos, gastos com viagens, despesas com festividades, além daquelas relacionadas com materiais de expediente. A composição dos fatores e sua ligação com as características de cada subelemento (Giacomoni, 2019), pode ser demonstrada pelo Quadro 2.

Quadro 2: Composição dos Fatores.

\begin{tabular}{|c|c|c|}
\hline Fatores & Subelementos & Características \\
\hline Fator 2 & $12,19,25,26,33,35,40,46,59$ & Bio, Exped, Manut, Lab, Fest \\
\hline Fator 3 & $04,07,10,20,21,22,24,29,36,43$ & Lab, Viag, Bio, Manut, Exped \\
\hline Fator 4 & $09,11,34,42$ & Lab, Manut \\
\hline Fator 5 & $16,44,49,54,58$ & Exped, Manut, Viag \\
\hline Fator 6 & $14,15,30,31$ & Bio, Fest, Exped \\
\hline
\end{tabular}

Nota: Bio = Materiais Biológicos; Exped = Materiais de Expediente; Manut = Manutenção de Máquinas e Equipamentos; Fest = Despesas com Festividades; Viag = Gastos com Viagens; Lab = Materiais de Laboratório.

Fonte: Autores (2020).

Percebe-se portanto, que os agrupamentos não respeitam de modo evidente as características naturais dos subelementos. Esse resultado indica que as despesas com materiais de consumo estão distribuídas sem alinhar o volume de gastos à sua característica. Como no caso das despesas com material de expediente que estão presentes em praticamente todos os fatores.

Ocorre que a execução orçamentária não permitiu distinguir o dispêndio de recursos de modo uniforme. Mesmo as características similares apresentaram gastos distintos. Algo que já foi observado desde as análises descritivas pela falta de normalidade. A falta de clareza nos agrupamentos indica a indissociabilidade entre grupos, inclusive nos períodos antes e após a adoção ao sistema eletrônico informatizado. Isto é, o argumento de redução nas despesas pode não prosperar para incentivar a aceitação de um sistema de informatização de processos eletrônicos, visto que a formação dos grupos não respeita as características naturais das despesas envolvidas. Entretanto, em que pese a incerteza acerca dos critérios para formulação dos fatores, os agrupamentos criados foram preservados para verificação da diferença entre grupos, consoante à segunda hipótese de pesquisa $H^{2}$.

Para realização do teste, primeiramente foi verificada a normalidade multivariada. Todavia, como demonstra a Tabela 3 , o pressuposto não foi atendido, razão pela qual se optou pela adoção ao teste de Kruskal-Wallis considerando " $k$ ” fatores independentes.

Tabela 3: Teste de Normalidade e Diferença de Médias.

\begin{tabular}{l|c|c|c}
\hline Normalidade Multivariada & \multicolumn{2}{|c}{$176.4366^{* * *}$} \\
\hline Mardia Skewness & & \multicolumn{2}{|c}{$6.7783^{* * *}$} \\
\hline Mardia Kurtosis & Normalidade Univariada & Assimetria & Curtose \\
\hline Fatores & 0.2112 & -0.3715 & -0.1593 \\
\hline Fator 2 & 0.1899 & -0.4600 & -1.0310 \\
\hline Fator 3 & 0.5010 & -0.0994 & -0.5973 \\
\hline Fator 4 & 0.1876 & 0.0295 & 0.8375 \\
\hline Fator 5 & $0.0387 * *$ & 0.3732 & -0.2519 \\
\hline
\end{tabular}

\section{Testes Não-Paramétricos de Diferença de Médias}

\begin{tabular}{ll}
\hline Teste de Kruskall- Wallis & $\chi^{2}(4)=1.2335, p$-valor $=0.8726$ \\
\hline
\end{tabular}

Nota: MS (Mardia Skewness), medida de assimetria; MK (Mardia Kurtosis), medida de curtose; Hipótese Nula para o teste de Normalidade Multivariada assume que $\mathrm{H}_{0}$ : a amostra provém de uma população com distribuição normal $\mathrm{N}(\mu, \sigma)$; Normalidade analisada a partir de Korkmaz, Goksuluk e Zararsiz (2014); Hipótese Nula para o Teste de Kruskall-Wallis H0: $\mu 1=\mu 2=\ldots=\mu \kappa$ (Fávero e Belfiore, 2017). 
Ao discutir os resultados frente às hipóteses de pesquisas, cumpre inicialmente destacar que também não foram encontrados argumentos para manutenção de $H^{2}$, dado o teste não-paramétrico de Kruskall-Wallis $(p$-valor $=0.8726$ ) que acusa a ausência de diferenciação entre grupos.

Este resultado demonstra que a aceitação do uso da tecnologia não remete à economicidade de despesas. Porventura, isso pode ser associado ao volume de despesas envolvidas, que dilui a percepção de economia. Outrossim, segundo referencial teórico (Giacomoni, 2019; ME, 2020), o impacto no implemento desse tipo de tecnologia é restrito aos materiais que deixam de ser utilizados, e que não foram significativos quando comparados às demais despesas.

Isso se mostra especialmente relevante num contexto em que a redução de despesas apresenta-se como um possível argumento em ambientes que mostram maior dificuldade em proporcionar mudanças em procedimentos. Portanto, haveria a possibilidade de economizar recursos, mantendo a agilidade e segurança na tramitação eletrônica processual, minimizando a resistência entre os atores envolvidos (Nowak, 2018; Fulton et al., 2018).

Apesar desse apelo que enaltece o discurso pela economicidade de despesas mediante o implemento do uso de tecnologia é preciso salientar que tanto as estatísticas descritivas, o teste de Wilcoxon consoante à $H^{l}$, bem como o teste de Kruskall-Wallis para verificação de $H^{2}$, não foram encontrados argumentos empíricos que demonstram a significância dessas medidas em decorrência da adoção ao sistema eletrônico informatização de processos.

Ocorre que, o volume de recursos despendidos pelo governo federal acaba por diluir a percepção de economicidade. Sobretudo quando a aplicação de sistemas eletrônicos informatizado para tramitação de processos apresenta reflexos quase somente às rotinas que são suprimidas com seu uso, no caso aquelas que fazem uso de papel e demais utensílios de escritório.

Ademais, é preciso ressaltar que, apesar de ter sido analisado o interstício de dois anos entre o implemento do sistema eletrônico informatizado, não foi observada uma mudança nos padrões das despesas públicas com material de consumo. Razão pela qual em $H^{2}$ não foi notada significância na distinção entre grupos, tão pouco a formação dos fatores pode ser explicada em contextos teóricos a partir do referencial que esclarece a características das rubricas orçamentárias da despesa pública (Giacomoni, 2019). Isto é, não foram encontradas evidências de que a economicidade de recursos, advinda com a adoção do sistema eletrônico informatizado, ocorre de modo distinto entre as naturezas de despesas.

Este resultado pode ser explicado pelo recorte orçamentário utilizado, composto pelas despesas de custeio (3), com gastos correntes (3), de aplicação direta (90), em materiais de consumo (30), que embora voltado à mesma rubrica das despesas de materiais de expediente (16), não expõe com a mesma nitidez o comportamento da variável representada neste subelemento (Giacomoni, 2019; Fulton et al., 2018).

Ao refutar $H^{1}$ e $H^{2}$, concomitantemente aos indicativos presentes nas estatísticas descritivas, descortina-se a possibilidade de utilização da economicidade de despesas como instrumento de aceitabilidade ao sistema eletrônico informatizado, visto que não se encontram a diferença no comportamento das despesas é homogênea entre os fatores analisados.

Todavia, ainda que as hipóteses de pesquisa não tenham sido sustentadas nos testes empíricos, há que se acentuar que os dispositivos teóricos permanecem intactos. Ou seja, ao verificar que o uso do sistema eletrônico informatizado não reflete numa diminuição representativa de despesas, acredita-se apenas que um argumento para aceitação do uso da tecnologia deixa de ser observado, neste caso.

Desta forma, as demais prerrogativas presentes na arguição teórica, amplamente debatida e testada nos estudos anteriores (Zhao; NI; Zhou, 2018; Mosw; Bwalya, 2018; Sherer; Teo, 2019; Carrera-Mora et al., 2019), ainda podem fomentar a aceitação e uso dos sistemas eletrônicos informatizados de tramitação e criação eletrônica de processos. Especialmente pela expectativa de melhorar o desempenho e esforço, a influência social e demais condições facilitadoras decorrentes do apelo por uma atuação governamental mais ágil, dinâmica, eficiente e efetiva. 
Diante dos achados da pesquisa, os benefícios pleiteados com a aceitação do uso de tecnologia, em especial quanto à economicidade de despesas, não se mostraram nítidos, embora, sejam percebidos na tramitação eletrônica online de dados e informações, represente uma maior visibilidade e transparência pública. Portanto, a partir desses resultados, não se pode incutir ao sistema eletrônico informatizado a prerrogativa de alteração nos padrões de despesas públicas. Ao passo que ainda persiste a maior segurança das informações, agilidade na tramitação eletrônica processual, acessibilidade online das informações e dinamismo na atuação governamental que são achados importantes para o processo de tomada de decisão dos gestores.

\section{Considerações Finais}

O presente estudo analisou a economicidade de despesas em instituições públicas visto a aceitação de tecnologia na informatização de processos eletrônicos. Para tanto, são utilizados fragmentos teóricos ligados à Aceitação e Uso de Tecnologia (UTAUT), que indicam a redução de despesas como um dos fatores ligados ao uso de ferramentas de tecnologia, além da segurança de dados e agilidade na tramitação de informações.

Como proxy de um sistema de informatização, foi utilizado o Sistema Eletrônico de Informações (SEI). O qual advém de um projeto para encontrar alternativas ao uso de processos físicos em órgãos públicos. O sistema oferece uma alternativa econômica às entidades aderentes. Todavia, em virtude ao volume de despesas executadas em todo âmbito federal, verificou-se a possibilidade de que a economia seja diluída e não se torne significativa.

Diante disso, surgiram duas hipóteses de pesquisa, a primeira aponta que a implementação de recursos tecnológicos oferece maior economicidade de recursos públicos. Já a segunda hipótese defende que a redução de despesas, advinda com a adoção do uso de sistema eletrônico informatizado, ocorre de modo distinto entre as naturezas de despesas.

Depois da análise, mediante estatísticas descritivas e testes de diferenças de médias, não foram encontrados argumentos que sustentem ambas hipóteses de pesquisa. Aceita-se que os demais aspectos levantados pelo componente teórico, preservam boa parte dos argumentos utilizados. Isso indica que o argumento de economia de despesas pode não ser apontado isoladamente para fomentar a adesão ao sistema eletrônico, mas precisa sustentar os demais benefícios advindos com o uso de tecnologia defendidos pela teoria de aceitação.

Ademais, a economia de recursos não ser significativa entre os períodos antes e depois da adoção ao sistema, não foram encontradas diferenças nas variâncias entre os diferentes grupos, indicando que as despesas se comportaram de modo assemelhado. Todavia, reitera-se que os demais aspectos levantados pelo componente teórico, preservam boa parte dos argumentos utilizados sobretudo quanto a agilidade, aprendizado, acessibilidade, facilidade do uso de tecnologia e segurança das informações tramitadas eletronicamente em tempo real.

A partir desses resultados entende-se que o discurso utilizado para incentivar a aceitação e uso da ferramenta precisa ser revisto, destacando os demais benefícios, principalmente, considerando a acessibilidade do usuário, criação de processo online e agilidade na tramitação eletrônica dos processos. Ou ainda, quantificar as mudanças de hábitos que podem trazer ganhos de eficácia, eficiência e efetividade, como defende a Nova Administração Pública. Diante desses apontamentos, sugere-se que estudos futuros verifiquem se a economicidade e os benefícios da adoção à tecnologia podem ser percebidos pelos usuários dos sistemas informatizados, identificando as perspectivas decorrentes da UTAUT que podem incentivar a aceitação da teoria e uso de tecnologia.

Além disso, recomenda-se ainda realizar uma análise comparativa entre antes e depois da implementação do sistema eletrônico informatizado, considerando no escopo do novo estudo, quando os processos administrativos eram totalmente físicos e muitas vezes de difícil acesso por parte dos usuários sedentos de informações em tempo hábil, dependo de autorizações nos locais de acesso restrito em que eram arquivados fisicamente os processos. 


\section{Referências}

Bhatiasevi, V. (2016). An extended UTAUT model to explain the adoption of mobile banking. Information Development. 32(4), $799-814$.

Cabrera-Sanchez, J-P., \& Villarejo-Ramos, Á. F. (2019). Fatores que Afetam a Adoção de Análises de Big Data em Empresas. Revista de Administração de Empresas. 59(6), 415-429.

Carrera-Mora, Ó., Ovando, C., Villafuerte, L., \& Parada, A. (2019). La relación de la perspectiva de eficiencia del ciudadano con su comportamiento de uso de los servicios de e-gobierno municipal. INNOVAR. 29(74), 133-146.

Fulton, D. C. P., Farias, J. S., Alfinito, S., \& Almeida, J. P. L. (2018). Adoção ao Sistema Eletrônico de Informações (SEI) pelo Ministério da Justiça: uma avaliação na ótica dos usuários. Revista do Serviço Público. 69(4), 1015 - 1036.

Giacomoni, J.(2019). Orçamento Governamental: teoria, sistema, processo. Atlas.

Gross, J., \& Ligges, U. (2015). Nortest: Tests for Normality. R package version1.0-4.

Gruzd, A., Staves, K., \& Wilk, A. (2012). Connected scholars: Examining the role of social media in research practices of faculty using the UTAUT model. Computers in Human Behavior. 28(6), 2340-2350.

Hair, J. F., Black, W. C., Babim, B. J., Anderson, R. E., \& Tatham, R. L. (2009). Análise Multivariada de Dados. (6a ed.), Bookman.

Korkmaz, S., Goksuluk, D., \& Zararsiz, G. (2014). MVN: An R Package for Assessing Multivariate Normality. The R Journal. 6(2), $151-162$.

Magsamen-Conrad, K., Upadhyaya, S., Joa, C. Y., \& Dowd, J. (2015). Bridging the divide: Using UTAUT to predict multigenerational tablet adoption practices. Computers in Human Behavior. 50(1), 186-196.

Maier, M. J. (2015). Companion Package to the Book “R: Einführung durch angewandte Statistik".Rpackage version0.9.3,<URL:http://CRAN.Rproject.org/package $=$ REdaS $>$.

Matias-Pereira, J. (2018). Administração Pública: foco nas instituições e ações governamentais. (5a ed.). Atlas.

ME. (2020). Processo Eletrônico Nacional. Ministério da Economia. <https://www.gov.br/economia/pt-br/assuntos/processo-eletronico-nacional>.

Mosweu, O., \& Bwalya, K. J. (2018). A multivariate analysis of the determinants for adoption and use of the Document Workflow Management System in Botswana's public sector. South African Journal of Libraries and Information Science. 84(2), 27-38.

Nowak, M. A. M. (2018). A Utilização do Sistema Eletrônico de Informações - SEI no Serviço Público: inovação tecnológica para melhoria na eficiência administrativa e no conhecimento institucional. Dissertação de Mestrado. Universidade Federal do Paraná. Setor de Ciências Sociais Aplicadas. Programa de Pós-Graduação em Desenvolvimento Econômico.

Ossani, P. C, \& Cirillo, M. A. (2020). MVar.pt: Analise multivariada. R package version 2.1.3.

Palos-Sanchez, P., Reyes-Menendez, A., \& Saura, J. R. (2019). Modelos de Adopción de Tecnologías de la Información y Cloud Computing en las Organizaciones. Información Tecnológica. 30(3), 3-12.

Purnama, D. E., Cahyono, A. D., \& Wijaya, A. F. (2020). Performance Analysis of Information System / Information Technology using COBIT 4.1 (PT. Global Infotech Solution). Journal of Information Systems and Informatics. 2(1), 105-113.

R Core Team. (2020). R: A language and environment for statistical computing. R Foundation for Statistical Computing, Vienna, Austria. URL https://www.Rproject.org/.

Sherer, R., \& Teo, T. (2019). Unpacking teachers' intentions to integrate technology: A meta-analysis. Educational Research Review. $27,90-109$.

Steccolini, I. (2019). Accounting and the post-new public management: Re-considering publicness in accounting research. Accounting, Auditing and Accountability Journal. 32(1), 255-279.

STN. (2020). Sistema Tesouro Gerencial. Ministério da Economia. Secretaria do Tesouro Nacional. <https://tesourogerencial.tesouro.gov.br /servlet/mstrWeb?pg=login>.

Venkatesh, V., Morris, M. G., Davis, G. B., \& Davis, F. D. (2003). User Acceptance of Information Technology: Toward a Unified View. MIS Quarterly. 27(3), $425-478$.

Zhao, Y., NI, Q., \& Zhou, R. (2018). What factors influence the mobile health service adoption? A meta-analysis and the moderating role of age. International Journal of Information Management. 43, 342-350. 